\title{
BOSQUES INUNDADOS EN LA AMAZONIA CENTRAL: SU APROVECHAMIENTO ACTUAL Y POTENCIAL. \\ Pia Parolin ${ }^{1}$
}

\section{Resumen.}

Las áreas boscosas de várzea en la Amazonia Central son periódicamente inundadas por ríos de aguas blancas ricos en sedimentos, que por lo tanto tienen suelos ricos y alta producción primaria. Han sido descritas 250 especies de árboles como típicas, muchas de ellas tienen baja densidad forestal. A pesar que la producción total de madera no es tan alta como en el bosque templado, los bosques de várzea han sido explotados desde finales del siglo pasado. La fácil accesibilidad y el alto número de individuos de una especie por área es ventajosa. Los recursos naturales como la producción de petróleo, jabón, resinas, fibras textiles, taninos, colorantes y medicinas, perfumes, látex y frutas son de importancia local y comercial. La madera de construcción tiene mucha importancia desde principios de siglo, cuando fue usada para la producción de energía en las embarcaiones y en la construcción civil y naval. La extracción de madera de construcción comenzó, a gran escala, en la década de los 20 . La extracción se realiza manualmente y la madera se transporta mediante en río hasta Manaus, donde la industria maderera está concentrada. El 80\% de la madera procesada permanece en el estado de Amazonas. El resto es exportada, principalmente al sur del Brasil, mientras que no hay comercio internacional. Las áreas inundadas son de excepcional importancia económica, para el cultivo de alimentos como para la crianza de ganado vacuno lechero, lo cual lleva a la destrucción de las áreas forestales. Muchas especies de árboles usadas comercialmente son desplazadas, especialmente en la cercanía de las grandes ciudades.

Palabras claves: Areas amazónicas inundadas, várzea, uso de madera de construcción y productos forestales, extracción.

\begin{abstract}
.
Central Amazonian whitewater floodplains (várzea) are periodically inundated by sediment rich rivers and therefore have rich soils and a high primary production. 250 tree species have been described as typical, many of which have low wood densities. Although total wood production is not higher than in temperate forests, várzea forests have been exploited since the end of the last century. The easy accessibility and the high number of individuals of a species per area are advantageous. Natural resources like the production of oil, soap, resins, textile fibres, tannins, colours and medicines, aromas, latex, and fruits are of local and commercial importance. Timber is a very important good since the beginning of the century, where it was used for energy production in steamboats, and for civil and naval construction. On a large scale the extraction of timber started in the 1920s. Logging is done by hand, and the wood is transported by raft to Manaus where sawmills and timber industry are concentrated. $80 \%$ of the processed wood remain in the State of Amazonas. The rest is exported mainly to Southern Brazil, while international trade is neglectable. The floodplains are of exceptional economic importance also for the cultivation of crops and cattle raising, which leads to the destruction of the forested areas. Many commercially used tree species are threatened, especially in the vicinity of big cities.
\end{abstract}

Key words: Central Amazonian floodplains, várzea, use of timber and forest products, logging.

\section{Introducción}

Las áreas boscosas de várzea en la Amazonia Central (Figura 1) son periódicamente inundadas por ríos de aguas blancas ricos en sedimentos, y poseen suelos más fértiles comparados con áreas inundadas por ríos de aguas negras, pobres en nutrientes (igapó), o tierra firme no inundada. La riqueza de los suelos permite una alta producción primaria; se manifiesta en la vegetación herbácea que cubre enormes áreas bajas, y en la cobertura densa y boscosa en las áreas más elevadas que tienen períodos de inundaciones cortos. Nuevas zonas de sedimentación son rápidamente

\footnotetext{
${ }^{1}$ Max-Planck-Institute for Limnology, Tropical Ecology, P.O. Box 165, 24302 Plön, Germany. Dirección postal actual: Universität Hamburg, Institut fur Allgemeine Botanik AB Systematik, Ohnhorststr. 18, 22609 Hamburg, Germany E-mail: piaparolin@gmx.de Tel. +49 / 40 / 8801007, Fax +49 / 40 / 88129282
} 
colonizadas por especies de árboles pioneros, de crecimiento rápido y de una secuencia sucesional de bosques altamente diversificados. Hoy en día, se conocen 250 especies de árboles como típicas para zonas de inundación por aguas blancas en diferentes sitios de la Amazonia (Worbes, 1997). La diversidad de especies varia dentro de la Amazonia. Inventarios florales señalan un gradiente de diversidad que crece desde el este al oeste. 53 especies $\mathrm{ha}^{-1}$ han sido descritas en un estudio realizado en la Amazonia Oriental (Ríos Guamá, Pará; Pires y Koury, 1959), 109 en la Amazonia Central (cerca de Manaus y Tefé; Ayres, 1993), y 149 en la Amazonia Occidental (región del Río Napo, Ecuador; Balslev et al., 1987). En la Amazonia Central el número de especies por hectárea es más bajo en áreas de bosques de aguas blancas que en las de aguas negras, donde han encontrado dos veces más especies $\mathrm{ha}^{-1}$ (Keel \& Prance, 1979; Worbes, 1986; Worbes, 1997).

Tabla 1: Especies seleccionadas de áreas inundadas por aguas blancas (várzea): densidad media de madera (con desvio padron) y incremento medio anual.

\begin{tabular}{|c|c|c|c|}
\hline Especie & $\begin{array}{c}\text { Estadio } \\
\text { sucesional }\end{array}$ & $\begin{array}{c}\text { Densidad de } \\
\text { madera } \\
{\left[\mathrm{g} \mathrm{cm}^{-3}\right]}\end{array}$ & $\begin{array}{c}\text { Incremen } \\
\text { to medio } \\
\text { anual } \\
{\left[\mathrm{mm} \mathrm{y}^{-1}\right]}\end{array}$ \\
\hline Cecropia latiloba & Pionera & $0.33 \pm 0.04$ & 15.0 \\
\hline Salix humboldtiana & Pionera & $0.39 \pm 0.01$ & - \\
\hline Senna reticulata & Pionera & $0.45 \pm 0.02$ & 21.4 \\
\hline Crateva benthami & $\begin{array}{c}\text { No } \\
\text { pionera }\end{array}$ & $0.49 \pm 0.05$ & 10.0 \\
\hline $\begin{array}{l}\text { Macrolobium } \\
\text { acaciifolium }\end{array}$ & $\begin{array}{c}\text { No } \\
\text { pionera }\end{array}$ & $0.49 \pm 0.07$ & 5.3 \\
\hline $\begin{array}{l}\text { Nectandra } \\
\text { amazonum }\end{array}$ & $\begin{array}{c}\text { No } \\
\text { pionera }\end{array}$ & $0.52 \pm 0.05$ & - \\
\hline Tabebuia barbata & $\begin{array}{c}\text { No } \\
\text { pionera }\end{array}$ & $0.65 \pm 0.02$ & 4.4 \\
\hline Vitex cymosa & $\begin{array}{c}\text { No } \\
\text { pionera }\end{array}$ & $0.56 \pm 0.06$ & 6.1 \\
\hline Crudia amazonica & $\begin{array}{c}\text { No } \\
\text { pionera }\end{array}$ & $0.87 \pm 0.05$ & 8.6 \\
\hline
\end{tabular}

\section{Resultados y discusión.}

En bosques de tierra firme de la Amazonia Central han sido descritas 65 a 176 especies ha ${ }^{-1}$ (Klinge \& Rodríguez 1968; Prance et al., 1976) y, al parecer, también en este ecosistema hay un gradiente de diversidad aumentando hacia los Andes, llegando hasta el número de 285 especies ha $^{-1}$ encontradas en la Amazonia Occidental (Balslev et al., 1987). La diversidad de especies es más alta en bosques no inundados, baja en bosques inundados por aguas negras, y más baja todavía en sistemas de aguas blancas. La distribución de las especies está confinada a los diferentes sistemas; en efecto, muchas de las especies comunes en zonas inundadas por aguas blancas están limitadas a aquellas áreas, pero no sucede en áreas inundados por aguas negras o bosques de tierra firme. Esto se puede explicar mediante las diferentes calidades de suelos, disponibilidad de nutrientes y condiciones hídricas del sistema (Prance, 1979; Kubilzki, 1989). En áreas inundadas por aguas blancas, las especies más citadas y esparcidas son Ceiba pentandra, Hura crepitans, Nectandra amazonum y Cecropia spp. (Worbes, 1997), es decir, especies de bosques de sucesión temprana, de baja densidad de madera $\left(0,25\right.$ a $0.62 \mathrm{~g} \mathrm{~cm}^{-3}$; Parolin \& Ferreira, 1998), y creciendo en áreas abiertas así como en bosques cerrados, pero limitadas a várzeas ricos y nutrientes.

Especies de baja densidad boscosa han sido encontradas como características para las inmediaciones de aguas blancas. La densidad de la madera en áreas inundadas por aguas blancas está entre 0,22 y $0,87 \mathrm{~g} \mathrm{~cm}^{-3}$ (Tabla 1); mientras que, en áreas inundadas por aguas negras es de 0,40 a 0,86 g $\mathrm{cm}^{-3}$. En bosques tropicales no inundados (tierra firme), la densidad de la madera es de 0.05 a $1.08 \mathrm{~g}$ $\mathrm{cm}^{-3}$ (Rueda \& Williamson, 1992).

El incremento anual de madera puede ser determinado a través del análisis de los anillos anuales de los árboles que se forman en muchas especies debido a la inundación regular (Worbes, 1984; Worbes \& Junk, 1989). Métodos dendrocronológicos para el cálculo del aumento de madera han sido usados e indican que las tasas de incremento cambian con las condiciones del hábitat y el estado de sucesión. Baja anchura del anillo por año (12,5 $\pm 6 \mathrm{~mm}$ por año) ha sido encontrada en especies de aguas blancas, comparado con $2,1 \pm 0,5 \mathrm{~mm}$ por año en especies de aguas negras. Especies de madera dura poseen tasas de incremento más bajas que especies de madera blanda. Diferencias interespecíficas han sido halladas en individuos de Tabebuia barbata que crecen en áreas inundadas por aguas blancas y negras, teniendo los anillos más del doble de ancho en individuos de aguas blancas (Parolin et al., 1998). Las diferencias en las tasas de incremento se podrían explicar a través de las variaciones de incremento según el estado de sucesión. Especies pioneras, de crecimiento rápido y de vida corta, que no se encuentran en áreas de aguas negras, poseen un promedio de $18,2 \pm 3 \mathrm{~mm}$ por año, $\mathrm{y}$ en las especies no pioneras de crecimiento lento y de vida larga el promedio es de $6,8 \pm 2 \mathrm{~mm}$ por año. Worbes (1997) asevera que, dependiendo de la edad de vegetación el incremento radial cambia. A nivel de comunidad en várzea una vegetación de 30 años posee un incremento medio de $9,9 \mathrm{~mm}$ por año y una vegetación con más de 200 años $1,7 \mathrm{~mm}$ por año. Por otro lado, el incremento radial de las vegetaciones no guarda relación directa con la producción de biomasa. Puede ser similar entre especies de etapas de sucesión diferentes, pero, en general, la productividad aumenta con la edad de vegetación. Una vegetación de Salix sp. de 2 años produce $1,5 \mathrm{t} \mathrm{ha}^{-1} \mathrm{y}^{-1}$, y vegetaciones más viejas pueden producir aún más (Worbes, 1997). En 
10 estudios hechos por Worbes, la producción promedia de madera en bosques tropicales mundialmente ha sido calculado en $7,3 \mathrm{t} \mathrm{ha}^{-1} \mathrm{y}^{-1}$, no excediendo la producción maderera de bosques templados. De esta manera, la producción maderera total de bosques tropicales no es muy alta, presumiblemente debido a altas pérdidas de respiración de la producción primaria neta total (Worbes, 1997). Los costos fisiológicos para el mantenimiento de la biomasa de los bosques aumentan con el peso del árbol. Aunque la producción primaria neta es muy alta, los bosques tropicales no representan el gran potencial para el uso sostenible y la silvicultura que nosotros hubiéramos esperado.

Muchas especies de árboles comunes poseen importancia comercial y han sido explotadas desde fines del siglo pasado. Arboles de áreas de inundación por aguas blancas son usados para la producción de aceites (p.e. Carapa guayanensis (Meliaceae), o palmeras como Oenocarpus bacaba y Astrocaryum jauari), jabón (Saboneteiro: Sapindus saponaria, Sapindaceae), resinas (generalmente especies de tierra firme como Hymenaea courbaril, Caesalpiniaceae o Protium spp., Burseraceae), fibras textiles (diferentes palmeras, p.e. Astrocaryum jauarí, cuyas hojas son usadas para la producción de fibras fuertes, o especies de la familia de Bombacaceae, como el miraguano (Kapok-tree), Ceiba pentandra), taninos (usados en el tratamiento de la piel), colores y medicinas (Le Cointe, 1922). Algunas especies de la familia Lauraceae son usadas en la producción de aromas. Entre las especies más importantes para el uso comercial, ambas de tierra firme y de áreas inundadas, están Hevea brasiliensis y $H$. spruceana (Euphorbiaceae), los árboles de caucho que producen látex. También muchos árboles producen frutos que son importantes en la dieta de peces y de los pueblos ribereños, o son usados para la exportación mundial (p.e. la Nuez de Brasil, Bertholletia excelsa, Lecythidaceae, en tierra firme).

Una mercancía muy importante para el uso local y la exportación es la madera. En el bosque de várzea que cubre aproximadamente 5,5 millones de hectáreas, existen $90 \mathrm{~m}^{-3} \mathrm{ha}^{-1}$ de comunidades de madera de construcción (Klenke \& Ohly, 1993). En Manaus, a comienzos del siglo, la madera fue usada para la producción de energía en los barcos de vapor y para construcciones civiles y navales. Originalmente, solo maderas con características y densidades específicas comparables a los bien conocidos árboles de Europa han sido explotados. Muchas maderas eran de tierra firme que por su alto peso específico garantizaron larga duración. "Itaúba" (Silvia tauba, Lauraceae), p.e., fue llamada "rois des bois" ("rey de los árboles") para la construcción naval, porque es de alta solidez (densidad de $0,94 \mathrm{~g} \mathrm{~cm}^{-3}$ ), fácil para trabajar y se pudre muy lento (Le Cointe, 1922). Por otro lado, muchos árboles comercialmente usados crecen en áreas inundadas donde el acceso fácil y el alto número de individuos de una especie por área son ventajas. Muchas especies de densidad baja son usadas por la gente ribereña en la construcción de casas flotantes, p.e. "Assacu" (Hura crepitans, Euphorbiaceae), un gran árbol típico de las zonas inundadas. Puesto que todo el árbol es aprovechado nunca es mencionado en estadísticas de aserraderos de Manaus, pero la densidad poblacional de estas especies está decreciendo rápidamente debido a la alta explotación local (Parolin, in press).

La extracción de madera de construcción a escala mayor en la Amazonia tan solo empezó en los años 1920, hasta 1918. Madera para Manaus era importada desde los Estados Unidos de América para la construcción de cajas, necesarias para el transporte y la exportación del caucho (Le Cointe, 1922). En 1900, $324.872 \mathrm{~kg}$. de madera de varias especies fueron exportados desde Pará. En 1920, el número aumentó a $40.000 .000 \mathrm{~kg}$. En comparación, solo $90.232 \mathrm{~kg}$. fueron exportados en 1919 desde el estado de Amazonas (Le Cointe, 1922). Hoy en día, mucha madera procede todavía de las áreas inundadas del río Amazonas, río Madeira y río Purus. El corte de leña se hace a mano y la madera es transportada en balsas a Manaus, dónde están concentrados los aserraderos y la industria maderera (Tabla 2; Klenke \& Ohly, 1993).

Tabla 2: Especies de árboles usados en $\operatorname{aserraderos}^{l}$ y en industria "veneer"2. Segundo Klenke et al. 1993.

\begin{tabular}{lc}
\hline Especie & Consumo [\%] \\
\hline Ocotea cymbarum $^{l}$ & 29.2 \\
Callophyllum brasiliense $^{l}$ & 25.0 \\
Marmaroxylon racemosum $^{l}$ & 12.4 \\
Diplotropis spp. $^{l}$ & 8.3 \\
Cedrela odorata $^{l}$ & 8.3 \\
Copaifera spp. $^{l}$ & 4.2 \\
Swietenia $_{\text {macrophylla }}{ }^{l}$ & 4.2 \\
Amburana cearensis $^{l}$ & 4.2 \\
Hura crepitans $^{1}$ & 4.2 \\
Ceiba pentandra $^{2}$ & 20 \\
Copaifera spp. $^{2}$ & 20 \\
Virola spp. $^{2}$ & 20 \\
Maquira sclerophylla $^{2}$ & 10
\end{tabular}

La explotación comercial de madera de baja densidad se ha incrementado. 25-30 especies de madera blanda que generalmente poseen un valor de mercado muy bajo son usadas, pero solo 12 de ellas son comercialmente significantes. Las especies típicas de la várzea son Virola surinamensis, Iryanthera surinamensis, Hura crepitans, Ceiba pentandra, Carapa guianensis, Calophyllum brasiliense, Copaifera sp., Naucleopsis caloneura, Pseudobombax munguba, Piranhea trifoliata, Hevea brasiliensis y Manilkara amazonica (Higuchi et al., 1994). Especies como Ceiba pentandra (Bombacaceae) son cultivadas en plantaciones para la producción de madera contrachapada. $80 \%$ de la madera procesada se queda en el estado de Amazonas. El resto es exportado, sobre 
todo al sur de Brasil, mientras el comercio internacional es descuidado (Klenke \& Ohly, 1993).

Debido a los suelos fértiles, las áreas inundadas por aguas blancas son de excepcional importancia económica. No solo la vegetación natural representa una muy alta productividad sino también es un ambiente muy bueno para el cultivo de plantas y la cría de ganado en pastos naturales o artificiales. En contraste con tierra firme, los bosques de áreas inundadas son de fácil acceso por barco, y el transporte de leña y frutas es facilitado por los ríos (Figura 2). La densidad poblacional humana aumenta todavía en la Amazonia, y la colonización de las áreas fértiles en zonas inundadas por aguas blancas está causando la destrucción de las tierras húmedas boscosas. Muchas especies de árboles comercialmente usadas están amenazadas, especialmente en la vecindad de grandes ciudades, y cada vez es más difícil de encontrar a bosques intactos en las áreas inmensas de las zonas inundadas de aguas blancas (Nepstad et al., 1999).

\section{Agradecimientos}

I wish to thank Jörg Ohly, Niro Higuchi and Angelica Vega Höring for their help with this manuscript. The field work in the floodplains was made possible by financial support from the INPA/Max-Planck Project, and by the assistance of the Instituto Nacional de Pesquisas da Amazônia (INPA).

\section{Referencias bibliográficas.}

Ayres J.M.C. 1993. As matas de várzea do Mamirauá. In: Estudos de Mamirauá. Sociedade Civil Mamirauá (ed.). : 1-123.

Balslev H., Lutteyn J., Yllgard B. \& Holm-Nielsen C. 1987. Composition and structure of adjacent unflooded and floodplain forest in Amazonian Ecuador. Opera Botanica. 92: 37-57.

Higuchi N., Hummel A.C., Freitas J.V., Malinowski J. R. y Stokes B.J. 1994. Exploraçao florestal nas várzeas do Estado do Amazonas: Seleçao de árvores, derrubada e transporte. Proceedings of the VIII Harvesting and Transportation of Timber Products Workshop. IUFRO / UFPr Curitiba, Paraná. : 168-193.

Keel S.H. \& Prance G.T. 1979. Studies of the vegetation of a white-sand black-water igapó (Rio Negro, Brazil). Acta Amazonica. 9: 645-655.

Klenke M. \& Ohly J.J. 1993. Wood from floodplains. In: 1st SHIFT Workshop, Belém, 1993. Junk W. J., Bianchi H. K. (eds.). GKSS-Research Center, Geesthacht. p. 88.

Klinge H. \& Rodrigues W. 1968. Litter production in an area of Amazonian terra firme forest. Part I. 1995)
Litterfall, organic and total nitrogen contents of litter. Amazoniana. 1: 287-302.

Kubitzki K. 1989. The ecogeographical differentiation of Amazonian inundation forests. Pl. Syst. Evol. 163: 285-304.

Le Cointe P. 1922. L'Amazonie brésilienne. Augustin Challamel (ed.), Librairie Maritime et Coloniale, Paris.

Nepstad D.C., Veríssimo A., Alencar A., Nobre C., Lima E., Lefebvre P., Schlesinger P., Potter C., Moutinho P., Mendoza E., Cochrane M. \& Brooks V. 1999. Large-scale impoverishment of Amazonian forests by logging and fire. Nature. 398: 505-508.

Parolin P., Ferreira L.V. \& Junk W.J. 1998. Central Amazonian floodplains: effect of two water types on the wood density of trees. Verh. Internat. Verein. Limnol. 26: 1106-1112.

Parolin P. \& Ferreira L.V. 1998. Are there differences in specific wood gravities between trees in várzea and igapó (Central Amazonia)?. Ecotropica. 4: 2532.

Parolin P. (In press). Growth, productivity and use of trees in white-water floodplains. In: Use and management of Amazonian floodplains. Junk W. J. (ed.).

Pires J.M. y Koury H.M. 1959. Estudo de um trecho de mata de Várzea proximo a Belem. 36: 3-44.

Prance G.T. 1979. Notes on the vegetation of Amazonia. III. Terminology of Amazonian forest types subjected to inundation. Brittonia, New York Botanical Garden. 31: 26-38.

Prance G.T., Rodrigues W.A. y da Silva M.F. 1976. Inventário florestal de um hectare de mata de terra firme $\mathrm{km} 30$ da estrada Manaus - Itacoatiara. Acta Amazonica. 6: 19-35.

Rueda R. \& Williamson G.B. 1992. Radial and vertical wood specific gravity in Ochroma pyramidale (Bombacaceae). Biotropica. 24: 512518.

Worbes M. 1986. Lebensbedingungen und Holzwachstum in zentralamazonischen Überschwemmungswäldern. Scripta Geobotanica. Erich Goltze. p. 112.

Worbes M. 1997. The forest ecosystem of the floodplains. In: The Central Amazon floodplain: Ecology of a pulsing system. Junk W. J. (ed.). Ecological Studies 126, Springer Verlag, Heidelberg. : 223-266.

Worbes M. \& Junk W.J. 1989. Dating tropical trees by means of $\mathrm{C} 14$ from bomb tests. Ecology. 70(2): 503-507.

Worbes M. 1984. Periodische Zuwachszonen an Bäumen zentralamazonischer Überschwemmungswälder. Naturwissenschaften. 71: 157-158. 